\title{
Variación estacional de la piritización de metales en sedimentos de un lagoon (laguna de Vixán - NO Península Ibérica)
}

\section{Seasonal variation of the metals piritization in sediments of a lagoon (Vixán lagoon - NW Iberian Peninsula)}

\author{
P. Fraga-Santiago1; X. L. Otero'; A. Pérez-Alberti ${ }^{2}$ \\ ${ }^{1}$ Departamento de Edafología y Química Agrícola, Facultad de Biología, USC. \\ Santiago de Compostela, España \\ pedro.fraga.santiago@gmail.com ${ }^{1}, \underline{x l . o t e r o @ u s c . e s ~}^{2}$
}

Departamento de Geografía, Facultad de Geografía e Historia, USC, Santiago de Compostela, España xepalber@usc.es

Recibido: Septiembre 2017 Aceptado: Noviembre, 2018

\section{RESUMEN}

La biodisponibilidad de los metales pesados ha sido ampliamente estudiada en los últimos años, debido fundamentalmente a su toxicidad para los ecosistemas naturales y para los seres vivos. En este trabajo, se estudió el efecto estacional sobre el proceso de piritización del $\mathrm{Fe}, \mathrm{Mn}$ y dos metales traza biolimitantes ( $\mathrm{Cu}$ y $\mathrm{Co}$ ) en los sedimentos de la laguna de Vixán (NO de la Península Ibérica). Los resultados muestran una intensa piritización del $\mathrm{Fe}$ y $\mathrm{Mn}$ (en general $>60 \%$ ) en los núcleos estudiados en ambas épocas del año 2011 (marzo y agosto). El Cu y Co presentaron un comportamiento diferente, mostrando una marcada variación estacional en superficie y una intensa piritización en profundidad $(>25 \mathrm{~cm})$.

Palabras clave: Pirita, Laguna, Sulfuro, metales traza, sedimento

\section{ABSTRACT}

The bioavailability of heavy metals has been extensively studied in recent years, mainly due to its toxicity to the natural ecosystems. In this work, it was studied the seasonal effect on the processes of pyritization of $\mathrm{Fe}, \mathrm{Mn}$ and two biolimitants trace metals ( $\mathrm{Cu}$ and $\mathrm{Co}$ ) in the sediments of the Vixán lagoon (NW Iberian Peninsula). The results show a strong pyritization of $\mathrm{Fe}$ and $\mathrm{Mn}$ (in general $>60 \%$ ) in the cores studied in both periods of the year 2011 (March and August). The $\mathrm{Cu}$ and $\mathrm{Co}$ showed a different behavior, showing a marked seasonal variation on surface and an intense pyritization below $25 \mathrm{~cm}$ depth.

Keywords: Pyrite, Lagoon, Sulfide, Trace metals, Sediment 


\section{INTRODUCCIÓN}

Los lagos costeros y las marismas son ecosistemas fuertemente amenazados dentro del contexto de cambio global actual ya que el incremento del nivel del mar va a suponer un cambio substancial en la configuración de estos hábitats de gran valor natural (Erwin, 2008), que a su vez son uno de los ecosistemas marinos más productivos, debido a su localización como medios de transición entre la tierra y el mar (Arienzo, y otros, 2014). En general, las lagunas costeras han sido caracterizadas por muchos autores como ecosistemas complejos y en frágil equilibrio, siendo entornos que tienen gran importancia ecológica y económica porque realizan funciones muy importantes albergando, refugiando y proporcionando alimento a gran variedad de especies continentales y marítimas muchas veces endémicas y vulnerables (Orkun, Galip, Demet, Yilmaz, \& Cagatayhan, 2010). De cualquier modo, la conservación de las funciones ecológicas de las lagunas pasa por mantener una buena calidad de las condiciones geoquímicas de las aguas y sedimentos que conforman las zonas intermareales en general y las lagunas litorales en particular, las cuales están sometidas a intensos cambios temporales relacionados fundamentalmente con variaciones en las condiciones meteorológicas y el movimiento mareal, que ejercen un fuerte control sobre el hidroperiodo (de Wit, y otros, 2001).

También es importante resaltar que en la actualidad debido a la densa humanización y a las actividades industriales y agrícolas que se realizan en las áreas de captación de lagos y lagunas, gran parte de estos ambientes se encuentran fuertemente antropizados presentando elevadas tasas de sedimentación y recibiendo aportes tanto de fuentes puntuales como difusas de diversos contaminantes como los metales traza; cuyo origen podría encontrase, entre otros, en los pesticidas y fertilizantes comúnmente utilizados en los campos agrícolas que rodean los lagos (Nicholson, Smith MCIWEM, Alloway, CarltonSmith, \& Chambers, 2006). Entre los contaminantes, los metales pesados causan una preocupación especial debido a sus propiedades no biodegradables y a sus efectos tóxicos cuando se acumulan en altas concentraciones en los organismos (Louma, 1983) (Jain, 2003) (Walling, y otros, 2003). Generalmente los metales que llegan a los ambientes acuáticos proceden de fuentes naturales como la meteorización de las rocas. Sin embargo, las actividades antrópicas como la agricultura y las actividades industriales y domésticas están incrementando las entradas de elementos tóxicos en estos ambientes receptores finales de aguas de escorrentía y de vertidos residuales (Förstner, 1979).

Los metales pueden llegar a la laguna de diversas formas (forma iónica, adsorbidos a coloides minerales y orgánicos, formando parte del material en suspensión, ...) siendo las formas solubles las más tóxicas (Jain, 2003) (Calmano, Hong, \& Förstner, 1993). La solubilidad de estos depende de las condiciones físico-químicas del agua y de los sedimentos. Por lo tanto, el conocimiento de los procesos biogeoquímicos que ocurren en las lagunas costeras es importante, ya que estos procesos pueden favorecer la disolución de metales, haciéndolos más o menos disponibles para la entrada en la cadena trófica. Por ello, el estudio geoquímico de estos medios procura presenciar cambios ambientales motivados por causas naturales o antropogénicas. Sin embargo, la geoquímica de las zonas húmedas muestra una dinámica altamente compleja, donde se producen cambios importantes en las condiciones geoquímicas del agua y de los sedimentos a pequeñas escalas temporales y espaciales (Otero \& Macías, 2003) (Otero \& Macías, 2002). Por ello, se pone de manifiesto que los estudios de este tipo de zonas húmedas deben contemplar una monitorización que recoja tanto los cambios estacionales como espaciales (Otero, Sánchez, \& Macías, 2000) (Otero \& Macías, 2003) (Otero \& Macías, 2002). 
Hasta la fecha, son escasos los estudios realizados en lagunas que consideren variaciones espaciotemporales y la mayoría de ellos se han centrado en la determinación de concentraciones totales de metales pesados en sedimentos. Sin embargo, estas mediciones quizás no proporcionen información adecuada para la evaluación del impacto y para la estimación de los efectos ambientales como la biodisponibilidad, movilidad y toxicidad de estos metales. En este sentido, el ciclo del $\mathrm{Fe}, \mathrm{Mn}$ y S es la clave en los procesos biogeoquímicos de los sedimentos costeros (Otero \& Macías, 2003) (Otero \& Macías, 2002) y puede determinar el comportamiento de los metales traza en estos ambientes (HuertaDíaz \& Morse, 1992) (Müller, 2002) (Charriau, y otros, 2011).

Actualmente, la especiación de metales en los sedimentos se propone para obtener información realista y adecuada y evaluar su incidencia ambiental (Quevauviller, y otros, 1997) (Davidson, Duncan, Littlejohn, Ure, \& Garden, 1998) (Sin, Chua, Lo, \& Ng, 2001). Los principios de los procedimientos de especiación se basan en la extracción selectiva de los metales usando los extractantes en el orden creciente de reactividad del metal para obtener una fracción sucesiva correspondiente a las formas de asociación metálicas (Tessier, Campbell, \& Bison, 1979) (Kersten \& Förstner, 1986). Sin embargo, a pesar del amplio número de publicaciones existentes relativas al estudio de metales pesados en suelos y sedimentos [(Merian \& Clarkson, 1991) (Adriano, 2001), entre otras] en la bibliografía no se ha encontrado demasiada información acerca de la geoquímica de los elementos traza en lagunas costeras. Teniendo esto presente, en este trabajo se planteó como principal objetivo el estudio de la dinámica temporal y con la profundidad de los sulfuros metálicos en la laguna de Vixán, una de las lagunas de mayor relevancia ambiental de Galicia. La pirita (FeS2) es un sumidero de metales traza en medios anóxicos (Huerta-Díaz \& Morse, Pyritization of trace metals in anoxic marine sediments, 1992). Sin embargo, la fracción metálica asociada a la pirita es una de las más dinámicas en los medios intermareales; estando sujeta tanto a procesos de síntesis, periodos de inundación prolongada, como de destrucción por oxidación, debido a cambios estacionales y/o bioperturbación (Otero, Sánchez, \& Macías, 2000) (Otero \& Macías, Spatial variation in pyritization of trace metals in salt-marsh soils, 2003) (Otero \& Macías, 2002), liberando los metales al agua intersticial y a la columna de agua. Por ello, en este trabajo se presentan los cambios estacionales en el centro de la laguna de la fracción pirítica del $\mathrm{Fe}, \mathrm{Mn}$ y de dos elementos biolimitantes como son el $\mathrm{Cu}$ y Co, realizando para ello una extracción secuencial que permite separar el metal cambiable, el metal asociado a los óxidos de Fe y el metal pirítico (Pulgar, 2004).

\section{MATERIAL Y MÉTODOS}

\subsection{Area de estudio}

La laguna de Vixán ( $\left.\mathrm{N} 42^{\circ} 32^{\prime} 22^{\prime \prime}, \mathrm{W} 9^{\circ} 1^{\prime} 27^{\prime \prime}\right)$ se encuadra dentro del Parque Natural del Complejo Dunar de Corrubedo y Lagunas de Carregal y Vixán situado en la costa suroeste de la provincia de A Coruña. Este espacio es muy importante desde el punto de vista económico y ecológico ya que proporciona un hábitat especial para diversas especies globalmente amenazadas [aves acuáticas y limícolas (Domínguez, Otero, \& Vidal, 2006)] en una de las rías de Galicia más productivas para la industria pesquera. La profundidad máxima de la laguna es de $(1,45) \mathrm{m}$ (Cillero, 2013) y su superficie aproximada es de 15 ha. Además, forma parte del conjunto de humedales costeros incorporados a la Red Natura 2000.

La laguna de Vixán muestra una forma irregular, siendo más ancha en la zona Oeste. La entrada de agua en la laguna es de carácter mixto: el aporte de 
agua dulce le llega a través de la lluvia, la escorrentía y dos tributarios de escasa entidad: uno en la zona Este y otro en la Norte (Rego de Vilar). Sin embargo, la entrada de agua salada de origen marino se produce a través de un canal que recorre la laguna en dirección Este-Oeste. Actualmente, el contacto con el mar no es permanente, limitándose al periodo invernal cuando las mareas vivas y las fuertes tormentas logran romper o superar la barrera arenosa que cierra su canal de desagüe. Además, en algunas ocasiones esta barra arenosa también es rota por los campesinos para favorecer el drenaje de la laguna y de las tierras de cultivo (Cillero, 2013).

\subsection{Toma de muestras y sedimentos}

Con el objetivo de establecer como afectan los cambios temporales de los suelos y sedimentos a las condiciones geoquímicas del sustrato se recogieron dos testigos (figura 1) en dos periodos distintos del año 2011: Uno a principios de primavera (marzo de 2011), cuando la laguna se encontraba totalmente llena y otro a mediados de verano (agosto de 2011), cuando la laguna se encontraba seca en más de $1 / 3$ de su superficie. Los núcleos de sedimento se tomaron en el centro de la laguna (figura 1). Esta zona, se caracteriza por presentar una lámina de agua permanente, excepto en años muy secos y estar colonizada, en parte, por plantas acuáticas como son: Ruppia maritima y Potamogeton pectinatus (Pulgar, 2004) (Cillero, 2013).

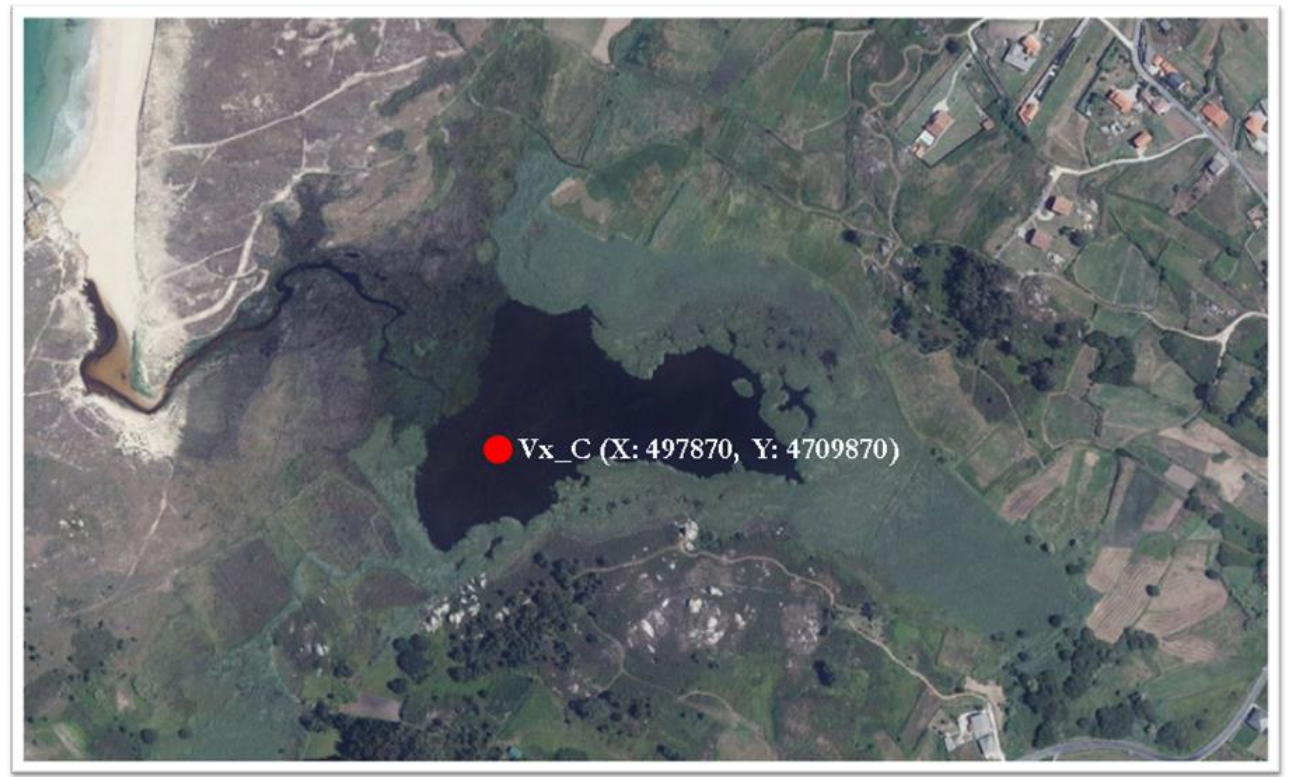

Figura 1. Localización del punto de muestreo en la laguna de Vixán (Fuente: Sixpac).

Los testigos se tomaron en tubos de PVC de $110 \mathrm{~cm}$ de largo y $4,5 \mathrm{~cm}$ de diámetro interno, que se cerraron herméticamente y se mantuvieron en posición vertical y refrigerados hasta la llegada al laboratorio, donde se guardaron en una cámara frigorífica a $3 \stackrel{\circ}{\circ}$. En menos de $24 \mathrm{~h}$, las muestras fueron extraídas y cortadas en sectores de 2 y $5 \mathrm{~cm}$ de espesor, dependiendo de los cambios morfológicos 0 texturales observados. Posteriormente, las muestras se mantuvieron congeladas a $-25^{\circ} \mathrm{C}$ hasta su análisis. 


\subsection{Análisis de las muestras de sedimentos}

Tanto el pH como el potencial redox (Eh) y la conductividad eléctrica (C.E.) se midieron "in situ", usando para ello electrodos específicos.

La extracción secuencial de Fe, Mn y metales traza se llevó a cabo a partir de 2 gramos de muestra húmeda (previamente homogenizada), para evitar alteraciones de las formas reducidas de $\mathrm{Fe}$ y $\mathrm{S}$ siguiendo un método combinado de (Tessier, Campbell, \& Bison, 1979), (Fortin, Leppard, \& Tessier, 1993) y (Huerta-Díaz \& Morse, 1990), ya aplicado en este departamento (Otero, Sánchez, \& Macías, 2000) (Otero \& Macías, 2002) (Otero, Vidal-Torrado, Calvo de Anta, \& Macías, 2005) (Otero, y otros, 2009) y que permite diferenciar las siguientes formas de Fe y metales asociados:

F1 $1_{\text {int: }}$ Fracción metálica soluble e intercambiable: Se añaden $30 \mathrm{ml}$ de una disolución $1 \mathrm{M}$ de $\mathrm{MgCl}_{2}$ con un $\mathrm{pH}$ de 7.0 y agitado durante 30 min (Tessier, Campbell, \& Bison, 1979). El sobrenadante se extrajo por centrifugado durante 30 min a $10000 \mathrm{rpm}$ y $4 \stackrel{\circ}{\circ}$, usando para ello una centrífuga refrigerada MPW 351R. Posteriormente, se realizó un lavado con agua Milli- $Q$ desoxigenada en $\mathrm{N}_{2}$ gas durante $15 \mathrm{~min}$. Estos dos últimos procesos (centrifugado y lavado) se repitieron en los pasos restantes que se describen a continuación:

F2 carb: Fracción metálica asociada a los carbonatos: $30 \mathrm{ml}$ de una disolución $1 \mathrm{M}$ de acetato de sodio con un $\mathrm{pH}$ de 5,0; agitado durante $5 \mathrm{~h}$ (Tessier, Campbell, \& Bison, 1979).

F3 oxi: Fracción metálica asociada a los óxidos e hidróxidos de Fe, tanto cristalinos (goethita y hematita) como amorfos (lepidocrocita, ferrihidrita): $30 \mathrm{ml}$ de una disolución de $0.35 \mathrm{M}$ de acetato $/ 0.2 \mathrm{M}$ de citrato de sodio con $1,5 \mathrm{~g}$ de ditionito de sodio a $\mathrm{pH} 4.8$ agitado continuamente durante 30 minutos a $75^{\circ} \mathrm{C}$ (Kostka \& Luther, 1994) (Kostka \& Luther, 1995).

F4 pir, Fracción metálica asociada a la pirita: $10 \mathrm{ml}$ de $\mathrm{HNO}_{3}$ concentrado. Las muestras se agitaron durante $2 \mathrm{~h}$. y luego fueron lavadas con 15 $\mathrm{ml}$ de agua Milli-Q que se incorporaron a la alícuota anterior considerándose un volumen final de $25 \mathrm{ml}$ (Huerta-Díaz \& Morse, 1990).

Antes de la extracción de la fracción pirítica, las muestras se sometieron a dos tratamientos: el primero con $30 \mathrm{ml}$ de HF $10 \mathrm{M}$ durante $16 \mathrm{~h}$ de agitación. Pasadas las $16 \mathrm{~h}$ se le añaden $3 \mathrm{~g}$ de Ac. Bórico y se agitan otras $8 \mathrm{~h}$ disolviéndose así los metales asociados a los aluminosilicatos. El segundo tratamiento se realiza con $10 \mathrm{ml}$ de $\mathrm{H}_{2} \mathrm{SO}_{4}$ concentrado durante 2 horas de agitación para eliminar los metales asociados a la materia orgánica (Huerta-Díaz \& Morse, 1992).

Tanto para la extracción secuencial como para la preparación de las disoluciones y el enjuague y lavado realizados durante los procedimientos experimentales se utilizó agua doblemente destilada Mili-Q. Además, el material de laboratorio empleado (recipientes y demás instrumentos de cristal y plástico) se sumergió en una disolución de $\mathrm{HNO}_{3}$ al $10 \%$ durante al menos 10 horas antes de su doble lavado con agua Mili-Q.

El metal reactivo (Mereactivo), la fracción de Fe que puede reaccionar con los sulfuros para formar pirita (Berner, 1970), fue extraído durante la F3oxi. El grado de piritización del Fe (DOP), término propuesto por Berner (Berner, 1970) o de los metales traza (DTMP) (Huerta-Díaz \& Morse, 1990), fue calculado con el objetivo de establecer el porcentaje de Mereactivo (principalmente óxidos e hidróxidos metálicos) incorporado a la fracción pirítica. El cálculo se hizo aplicando la ecuación (1) y asumiendo que el Mereactivo se extrae con ditionito de sodio (DOP- 
ditionito) de acuerdo con las recomendaciones de Raiswell (Raiswell, Canfield, \& Berner, 1994):

$$
\operatorname{DOP} / D T M P(\%)=\left(\frac{M e \text { pirítico }}{M e_{\text {reactivo }}+\text { Me pirítico }}\right) \cdot 100(1)
$$

\section{RESULTADOS Y DISCUSIÓN}

\subsection{Características generales de los sedimentos.}

El pH presentó valores ligeramente alcalinos, oscilando entre $7.4(5 \mathrm{~cm}$ de profundidad, marzo 2011) y 7.8 . (47.5 $\mathrm{cm}$ de profundidad, marzo 2011). El potencial redox durante el mes de marzo osciló entre $-106 \mathrm{mV}$ y $-76 \mathrm{mV}$, lo que indica que las condiciones del sedimento son fuertemente reducidas ya en superficie, a pesar de presentar una lámina de agua óxica $(E h>450 \mathrm{mV}$, datos no mostrados). La conductividad eléctrica del agua (valores todavía no publicados) mostró una marcada estacionalidad mostrando los valores más elevados en verano (laguna parcialmente seca). La conductividad eléctrica de las muestras superficiales del sedimento refleja la fuerte salinidad del ambiente. Estos valores oscilaron entre $3.73 \mathrm{dS} / \mathrm{m}$ (47.5 cm de profundidad, marzo 2011) y $17.45 \mathrm{dS} / \mathrm{m}$ (12.5 cm de profundidad, marzo 2011). Así, estos resultados obtenidos ponen de manifiesto que las aguas de la laguna tienen un origen mixto (marino y continental).

\subsection{Variación, estacional y con la profundidad de la piritización de metales.}

La composición y dinámica geoquímica del sustrato en las zonas húmedas depende de múltiples factores como son: entorno geológico, clima, actividad biológica y actividad humana (Cox \& Preda, 2005). Finalmente, la biodisponibilidad, la removilización de los metales y su transferencia a la cadena trófica puede verse afectada por cambios en las condiciones ambientales como el $\mathrm{pH}$, el potencial redox, la salinidad, las concentraciones y el tipo de ligando y de agentes quelantes presentes en los sedimentos (Jain, 2003) (Förstner, Ahlf, \& Calmano, 1989) (Calmano, Hong, \& Förstner, 1993).

En general, la piritización de metales con la profundidad no presentó un patrón claro observándose incrementos y disminuciones irregulares a profundidades intermedias (figura 2).

\section{Grado de piritización de Fe}

El grado de piritización (DOP) es un indicador geoquímico del estado redox de las aguas profundas (Raiswell \& Canfield, 2012). Como se observa en la (figura 2) el porcentaje de $\mathrm{Fe}$ piritizado en el mes de marzo varía irregularmente con la profundidad desde el $69 \%$ a los $5 \mathrm{~cm}$ hasta llegar al $91 \%$ a los $42.5 \mathrm{~cm}$. Además, en el periodo estival se presenta un comportamiento similar al de principios de primavera, ya que el porcentaje de Fe piritizado también va aumentando con la profundidad hasta llegar al $92 \%$, también a los $42,5 \mathrm{~cm}$. Los elevados valores de DOP encontraros en este estudio son propios de ambientes sedimentarios pobremente oxigenados (DOP: $55-75 \%$ ) o de aguas profundas euxínicas 
(DOP $>75 \%$ ) (Botsou, y otros, 2015). Por otra banda, valores elevados de DOP también se presentan en marismas (Rouychoudhury, Kostka, \& Van Cappellen, 2003) y lagos costeros (Neumann, y otros, 2005) en los que la deposición de sedimentos ocurre bajo aguas estrictamente oxigenadas. En estos entornos, enterramientos físicos y biológicos intensos y pequeños cambios del potencial redox del sedimento pueden actuar reciclando, removilizando y concentrando localmente el Fe lábil en el interior de los sedimentos (Lyons \& Severmann, 2006). Además, en las capas profundas de sedimento, que no son susceptibles de bioturbación, la piritización intensa puede ocurrir a través del incremento de la fracción pirítica, por ejemplo a través de la adsorción de complejos acuosos de Fe$\mathrm{S}-\mathrm{H}$, formados inicialmente en la capa superficial, en los cristales piríticos preexistentes (Kostka \& Luther, 1995). En los resultados obtenidos a partir de los 42, $5 \mathrm{~cm}$, el DOP decrece (35\%) para volver a aumentar irregularmente a los $72.5 \mathrm{~cm}$ de profundidad (55\%). Los porcentajes de piritización del mes de agosto (laguna parcialmente seca) a los 2,5 y a los $12,5 \mathrm{~cm}$ de profundidad son entre un 10 y un $20 \%$ inferiores a los obtenidos en el mes de marzo (laguna totalmente llena). Sin embargo, a los $22.5 \mathrm{~cm}$ de profundidad la situación se invierte y los porcentajes de piritización de agosto son prácticamente un $50 \%$ mayores a los de marzo.

En general, estos elevados porcentajes de piritización (DOP $>50 \%$ ) están en consonancia con las condiciones fuertemente reducidas del sedimento $(\mathrm{EH}<0$ $\mathrm{mV})$, que se mantuvieron durante todo el año.

\section{Grado de piritización del Mn}

El Mn presentó elevados porcentajes de piritización (DTMP) en ambas estaciones, superiores incluso a los del Fe (figura 2). Trabajos previos muestran que el Mn comienza su piritización a partir de un DOP > 40\% y no suele superar la piritización del Fe [ver por ejemplo (Huerta-Díaz \& Morse, 1990) (Huerta-Díaz \& Morse, 1992) (Morse \& Luther III, 1999) (Otero \& Macías, Variation with deph and season in metal sulfides in salt marsh soils, 2002)]. Además, en estos resultados, al igual que en el caso del Fe, el porcentaje de Mn piritizado en el mes de marzo va aumentando irregularmente con la profundidad desde el $61 \%$ a los $5 \mathrm{~cm}$ hasta llegar al $80 \%$ a los $42.5 \mathrm{~cm}$. En el mes de agosto se presenta un comportamiento similar al de marzo, aunque con porcentajes de Mn piritizado en todas las muestras ligeramente superiores a los del muestreo anterior, el porcentaje de $\mathrm{Mn}$ pirítico también va aumentando con la profundidad hasta llegar al $92 \%$ a los 42,5 $\mathrm{cm}$. Todos los porcentajes de piritización obtenidos para el $\mathrm{Mn}$ en el periodo estival (laguna parcialmente seca) son superiores a los obtenidos en el mes de marzo (laguna totalmente llena) debido a que al aumentar las concentraciones de las fracciones fácilmente oxidables encontradas en el sedimento en verano, también aumentan los porcentajes de piritización.

\section{Grado de piritización de Cu y Co}

El Cu y el Co presentaron un patrón de comportamiento más complejo que el Fe y el Mn. Ambos elementos presentan en general una elevada piritización (DTMP), alcanzando por debajo de los $25 \mathrm{~cm}$ de profundidad a principios de primavera valores superiores al $70 \%$ en el caso del Cu y al $90 \%$ para el Co (figura 2). No obstante, a diferencia del $\mathrm{Fe}$ y $\mathrm{Mn}$, el $\mathrm{Cu}$ y $\mathrm{Co}$ mostraron una fuerte disminución del DOP en superficie para el periodo estival. 
En general, la piritización de $\mathrm{Cu}$ y Co fue similar o superior al DOP (\%) debido a que la cinética de reacción de ambos metales con el sulfuro es superior a la del Fe (Morse \& Luther III, 1999). Sin embargo, en superficie mostraron una variabilidad estacional mayor a la del $\mathrm{Fe}$, presumiblemente porque los sulfuros mixtos son menos estables que las formas puras y por tanto más sensibles a los cambios estacionales que la propia pirita. No obstante, este aspecto debe ser objeto de un estudio más detallado (Faber, Lukowski, Ding, Kaiser, \& Jin, 2014).

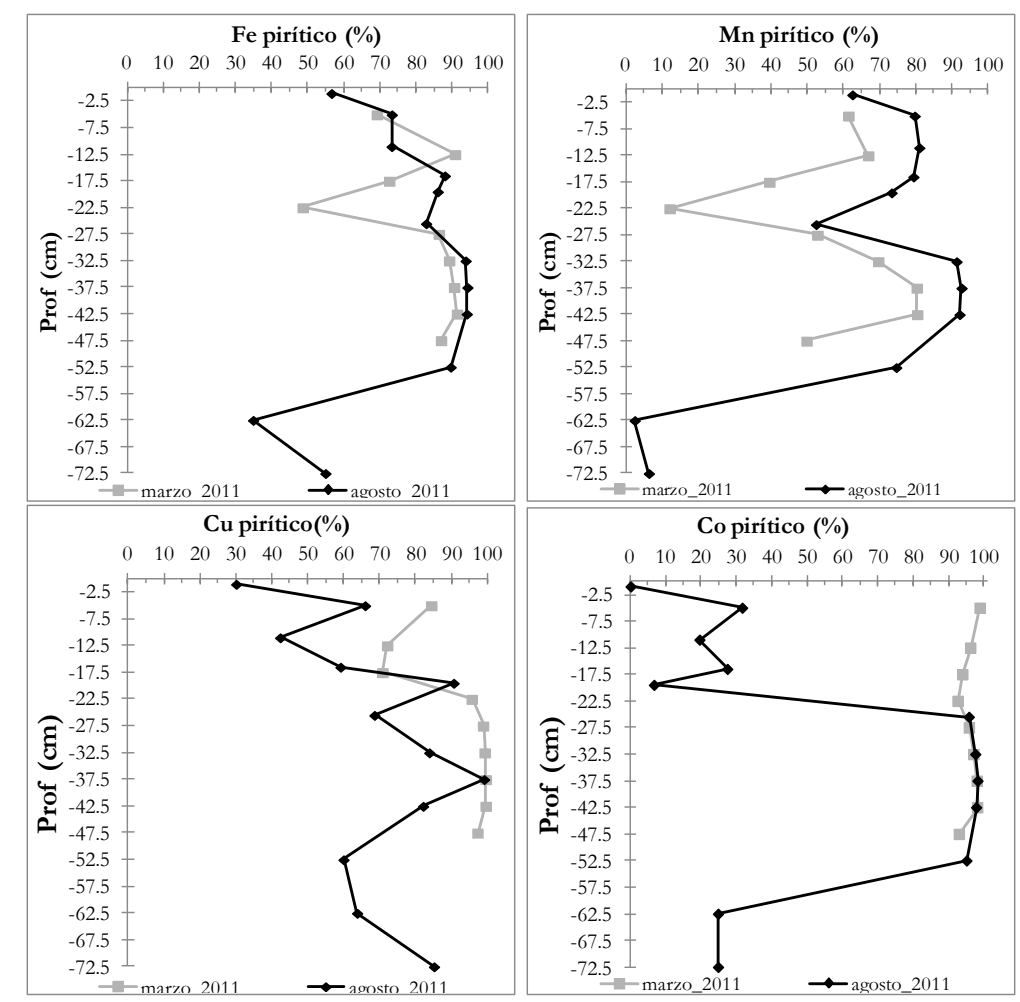

\section{Figura 2. Variación estacional y con la profundidad del grado de piritización de metales en la laguna de Vixán.}

\section{CONCLUSIONES}

Los sedimentos del centro de la Laguna de Vixán, independientemente de la estación del año, muestran condiciones fuertemente reducidas en todo su perfil propias casi de medios euxínicos, a pesar de presentar una lámina de agua con condiciones óxicas. En consonancia con las condiciones Eh-pH, el Fe y el Mn se encuentran fundamentalmente asociados a la fracción pirítica; mientras que el $\mathrm{Cu}$ y Co muestran un comportamiento geoquímico mucho más complejo, con fuertes variaciones estacionales que deben ser estudiadas con más detalle en futuros trabajos. 


\section{REFERENCIAS}

1 Adriano, D. C. (2001). Trace Elements in Terrestrial Environments. Springer.

2 Arienzo, M., Toscano, F., Di Fraia, M., Caputi, L., Sordino, P., Guida, M., . . . Ferrara, L. (2014). An assessment of contamination of the Fusaro Lagoon (Compania Province, southern Italy) by trace metals. Environ Monit Assess, 186, 5731-5747.

3 Berner, R. A. (1970). Sedimentary pyrite formation. American Journal of Science, 268, 1-23.

4 Botsou, F., Godelitsas, A., Kaberi, H., Mertzimekis, T. J., Goettlicher, J., \& Scoullos, M. (2015). Distribution and partitioning of major and trace elements in pyrite-bearing sediments of a Mediterranean coastal lagoon. Chemie der Erde, 75, 219-236.

5 Caille, N., Tiffreau, C., Levyal, C., \& Morel, J. L. (2003). Solubility of metals in an anoxic sediment during prolonged aeration. Science of Total Environment, 301(1-3), 239-250.

6 Calmano, W., Hong, J., \& Förstner, U. (1993). Binding and remobilisation of heavy metals in contaminated sediment affected by $\mathrm{pH}$ and redox potential. Water Science and Technology, 28, 223-235.

7 Charriau, A., Lesven, L., Gao, Y., Leermakers, M., Baeyens, W., Ouddane, B., \& Billon, G. (2011). Trace metal behaviour in riverine sediments: role of organic matter and sulphides. Applied Geochemistry, 26, 80-90.

8 Cillero, C. (2013). Identificación y definición del estado de conservación de humedales lagunares de Galicia y su intengración en el sistema territorial. Tesis Doctoral. Universidad de Santiago de Compostela.

9 Cox, M. E., \& Preda, M. (2005). Trace Metal Distribution Within Marine and Estuarine Sediments of Western Moreton Bay, Queensland, Australia: Relation to Land Use and Setting. Geographical Research, 43(2), 173-193.

10 Davidson, C. M., Duncan, A. L., Littlejohn, D., Ure, A. M., \& Garden, L. M. (1998). A critical evaluation of the three-stage BCR sequential extraction procedure to assess the potential mobility and toxicity of heavy metals in industrially-contaminated land. Analytica Chimica Acta, 363, 45-55.

11 de Wit, R., Stal, L. J., Lomstein, B. A., Herbert, R. A., van Gemerden, H., Viaroli, P., . . . Heijs, S. K. (2001). ROBUST: The ROle of BUffering capacities in STabilising coastal lagoon ecosystems. Continental Shelf Research, 21, 2021-2041.

12 Domínguez, J., Otero, M., \& Vidal, M. (2006). Guía de las aves del Parque Natural del Complejo Dunar de Corrubedo y Lagunas de Carregal y Vixán. Xunta de Galicia.

13 Eggleton, J., \& Thomas, K. V. (2004). A review of factors affecting the release and bioavailability of contaminants during sediment disturbance events. Environment International, 30, 973-980.

14 Erwin, K. L. (2009). Wetlands and global climate change: the role of wetland restoration in a changing world. Wetlands Ecol Manage, 17, 71-84.

15 Faber, M. S., Lukowski, M. A., Ding, Q., Kaiser, N. S., \& Jin, S. (2014). Earth-Abundant Metal Pyrites (FeS2, CoS2, NiS2, and Their Alloys) for Highly Efficient Hydrogen Evolution and Polysulfide Reduction Electrocatalysis. The journal of Physical Chemestry, 118, 21347-21356. 
16 Filgueiras, A. V., Lavilla, I., \& Bendicho, C. (2004). Evaluation of distribution, mobility and binding behaviour of heavy metals in surficial sediments of Louro River (Galicia, Spain) using chemometric analysis: a case study. Science of Total Environment, 330, 115-129.

17 Förstner, U. (1979). Sources and sediment associations of heavy metals in polluted coastal regions. Physics and Chemistry of the Earth, 11, 849-866.

18 Förstner, U., Ahlf, W., \& Calmano, W. (1989). Studies on the transfer of heavy metals between sedimentary phases with a multi-chamber device: combined effects of salinityand redox potential. Marine Chemistry, 28, 145158.

19 Fortin, D., Leppard, G., \& Tessier, A. (1993). Characteristic of lacustrine diagenic iron oxyhydroxides. Geochimica et Cosmochimica Acta, 57, 43914404.

20 Huerta-Díaz, M. A., \& Morse, J. W. (1990). A quantitative method for determination of trace metal concentrations in sedimentary pyrite. Marine Chemistry, 29, 119-144.

21 Huerta-Díaz, M. A., \& Morse, J. W. (1992). Pyritization of trace metals in anoxic marine sediments. Geochimica et Cosmochimica Acta, 56, 26812702.

22 Jain, C. K. (2003). Metal fractionation study on bed sediments of River Yamuna, India. Water Research, 38, 569-578.

23 Kersten, M., \& Förstner, U. (1986). Chemical fractionation of heavy metals in anoxic estuarine and coastal sediments. Water Science and Technology, 18, 121-130.

24 Kostka, J. E., \& Luther, I. G. (1994). Partitioning and speciation of soil phase iron in saltmarsh sediments. Geochimica et Cosmochimica Acta, 58, 1701-1710.

25 Kostka, J. E., \& Luther, I. G. (1995). Seasonal cycling of Fe in saltmarsh sediments. Biogeochemistry, 29, 159-181.

26 Louma, S. N. (1983). Bioavailability of trace metals to aquatic organism-a review. Science of Total Environment, 28, 1-22.

27 Lyons, T. W., \& Severmann, S. (2006). A critical look at iron paleoredox proxies: newinsights from modern euxinic marine basins. Geochimica et Cosmochimica, 70, 5698-5722.

28 Merian, E., \& Clarkson, T. (1991). Metals and Their Compounds in the Environment: Occurrence, Analysis, and Biological Relevance. $\mathrm{VCH}$.

29 Morse, J. W., \& Luther III, G. W. (1999). Chemical influences on trace metal-sulfide inter-actions in anoxic sediments. Geochimica et Cosmochimica, 63, 3373-3378.

30 Müller, A. (2002). Pyritization of iron and trace metals in anoxic fjord sediments (Nordåsvannet fjord, Western Norway). Applied Geochemistry, 17, 923-933.

31 Neumann, T., Rausch, N., Leipe, T., Dellwig, O., Berner, Z., \& Böttcher, E. (2005). Intensepyrite formation under low-sulfate conditions in the Achterwasser lagoon, SW Baltic Sea. Geochimica et Cosmochimica, 69, 3619-3630.

32 Nicholson, F. A., Smith MCIWEM, S. R., Alloway, B. J., Carlton-Smith, C., \& Chambers, B. J. (2006). Quantifying heavy metal inputs to agricultural soils in England and Wales. Water and Environment Journal, 20, 87-95. 
33 Orkun, I. D., Galip, S., Demet, G. K., Yilmaz, T., \& Cagatayhan, B. E. (2010). Speciation and implications of heavy metal content in surface sediments of Akyatan Lagoon-Turkey. Desalination, 260, 199-210.

34 Otero, X. L., \& Macías, F. (2002). Variation with deph and season in metal sulfides in salt marsh soils. Biogeochemistry, 61, 247-268.

35 Otero, X. L., \& Macías, F. (2003). Spatial variation in pyritization of trace metals in salt-marsh soils. Biogeochemistry, 62, 59-86.

36 Otero, X. L., Ferreira, T. O., Huerta-Díaz, M. A., Partiti, C., Souza Jr, V., Vidal-Torrado, P., \& Macías, F. (2009). Geochemistry of iron and manganese in soils and sediments of a mangrove system, Island of Pai Matos (Cananeia - SP, Brazil). GeodermaGeoderma, 148, 318-335.

37 Otero, X. L., Sánchez, J. M., \& Macías, F. (2000). Bioaccumulation of heavy metals in thionic fluvisols by a marine polychaete (Nereis diversicolor): The role of metal sulfide. Journal of Environmental Quality, 29, 1133-1141.

38 Otero, X. L., Vidal-Torrado, P., Calvo de Anta, R., \& Macías, F. (2005). Trace elements in biodeposits and sediments from musell culture in the ría de Arousa (Galicia-NW Spain). Environmental Pollution, 136, 119-134.

39 Pulgar, I. (2004). Guía de la flora del Parque Natural del Complejo Dunar de Corrubedo y Lagunas de Carregal y Vixán. Xunta de Galicia.

40 Quevauviller, P., Rauret, G., Lopez-Sanchez, J. -F., Rubio, R., Ure, A., \& Muntau, H. (1997). Certification of trace metal extractable contents in a sediment reference material (CRM 601) following a three-step sequential extraction procedure. The Science of the Total Environment, 205, 223-234.

41 Raiswell, R., \& Canfield, D. E. (2012). The iron biogeochemical cycle past and present. Geochemical Perspectives.European Association of Geochemistry., 1(1).

42 Raiswell, R., Canfield, D. E., \& Berner, R. A. (1994). A comparasion of iron extraction methods of degree of pyritisation and the recognition of ironlimited formation. Chemical Geology, 111, 101-110.

43 Rouychoudhury, A. N., Kostka, J. E., \& Van Cappellen, P. (2003). Pyritization: a palaeoenvironmental and redox proxy reevaluated. Estuarine Coastal and Shelf Science, 57, 1183-1193.

44 Salomons, W., Kerdlik, H., van Pagee, H., Klomp, R., \& Schreur, A. (1988). Behaviour and Impact Assessment of Heavy Metals in Estuarine and Coastal Zones, in: U. Seeliger, L.D. de Lacerda, S.R. Patchineelam (Eds.), Metals in Coastal Environments of Latin America. New York: SpringerVerlag.

45 Scholz, F., \& Neumann, T. (2007). Trace element diagenesis in pyrite-rich sediments of the Achterwasser lagoon, SW Baltic Sea. Marine Chemistry, 107, 516-532.

46 Shikazono, N., Nakata, M., \& Tokuyama, E. (1994). Pyrite with high Mn content from the Nankai Trough formed from subduction-induced cold seepage. Marine Geology, 118, 303-313.

47 Sin, S. N., Chua, H., Lo, W., \& Ng, L. M. (2001). Assessment of heavy metal cations in the sediments of Shing Mun River, Hong Kong. Environment International, 26, 297-301.

48 Tessier, A., Campbell, P., \& Bison, M. (1979). Sequential extraction procedure for the speciation of particulate trace metals. Analytic Chemistry, 51, 844-851. 
49 Walling, D. E., Owens, P. N., Carter, J., Leeks, G. J., Lewis, S., Meharg, A. A., \& Wright, J. (2003). Storage of sediment-associated nutrients and contaminants in river channel and floodplain systems. Applied Geochemistry, 18, 195-220.

50 Zoumis, T., Schmidt, A., Grigorova, L., \& Calmano, W. (2001). Contaminants in sediments: remobilisation and demobilisation. Science of Total Environment, 266, 195-202. 